\title{
Remission of acanthosis nigricans after treatment with metformin in a teenager
}

Tsiroukidou $\mathrm{K}^{1}$, Zografou $\mathrm{I}^{2}$, Griva $\mathrm{T}^{2}$, Pyrpasopoulou $\mathrm{A}^{2}$, Karagianni $\mathrm{P}^{1}$, Papagianni $\mathrm{M}^{1}$, Kosta $\mathrm{K}^{1}$, Vamvakis $\mathrm{A}^{1}$, Efstathiadou $\mathrm{Z}^{3}$, Sampanis $\mathrm{C}^{2}$

3rd Pediatric Department Aristotle University of Thessaloniki ${ }^{1}$, Diabetes Center ${ }^{2}$, Department of Endocrinology ${ }^{3}$, Hippokrateion General Hospital Of Thessaloniki

Introduction: Acanthosis nigricans is a condition commonly associated with disorders characterized by insulin resistance. Data regarding the treatment of acanthosis nigricans are still insufficient.

Case report: A 10- year old overweight girl $(B M I=24 \mathrm{~kg} / \mathrm{m} 2)$ presented at the obesity outpatient clinic. Her family history was negative for metabolic syndrome. Clinical examination revealed extensive acanthosis nigricans involving the neck and armpit. Laboratory tests were normal apart from high insulin levels related to glucose levels, that confirmed insulin resistance (HOMA-IR=14) (Table 1). The patient followed hypocaloric diet and exercise and had no clinical improvement one year after. Acanthosis nigricans expanded involving neck, armpit and thorax (Figure 1A), as insulin resistance index increased (HOMA-IR=28.6). Metformin at a dose of $1700 \mathrm{mg}$ daily was added to the diet and exercise with good clinical response. Insulin resistance decreased significantly (HOMA-IR=5.85) and acanthosis nigricans improved during the next 12 months (Figure 1B). Moreover there was improvement in OGTT during the follow-up period (Table 2).

\begin{tabular}{|c|c|c|c|c|c|}
\hline Time (min) & 0 & 30 & 60 & 90 & 120 \\
\hline \multicolumn{6}{|c|}{9,5 years } \\
\hline $\begin{array}{l}\text { Blood Glucose } \\
\text { (mg/dl) }\end{array}$ & 93 & 150 & 147 & 109 & 110 \\
\hline Insulin ( $\mu \mathrm{UI} / \mathrm{ml})$ & 62,9 & 626,4 & 759,4 & 394,3 & 396,5 \\
\hline \multicolumn{6}{|c|}{10,5 years } \\
\hline $\begin{array}{l}\text { Blood Glucose } \\
\text { (mg/dl) }\end{array}$ & 88 & 137 & 134 & 109 & 122 \\
\hline Insulin ( $\mu \mathrm{UI} / \mathrm{ml})$ & 132,0 & 469,0 & 546,0 & 470,0 & 530,0 \\
\hline \multicolumn{6}{|c|}{11,5 years } \\
\hline $\begin{array}{l}\text { Blood Glucose } \\
\text { (mg/dl) }\end{array}$ & 80 & 159 & 153 & 138 & 119 \\
\hline Insulin ( $\mu \mathrm{UI} / \mathrm{ml})$ & 29,6 & 300,0 & 300,0 & 281,0 & 80,8 \\
\hline \multicolumn{6}{|c|}{13 years } \\
\hline $\begin{array}{l}\text { Blood Glucose } \\
\text { (mg/dl) }\end{array}$ & 81 & 159 & 167 & 138 & 125 \\
\hline Insulin ( $\mu \mathrm{UI} / \mathrm{ml})$ & 11,2 & 130,0 & 116,0 & 110,0 & 94,9 \\
\hline
\end{tabular}

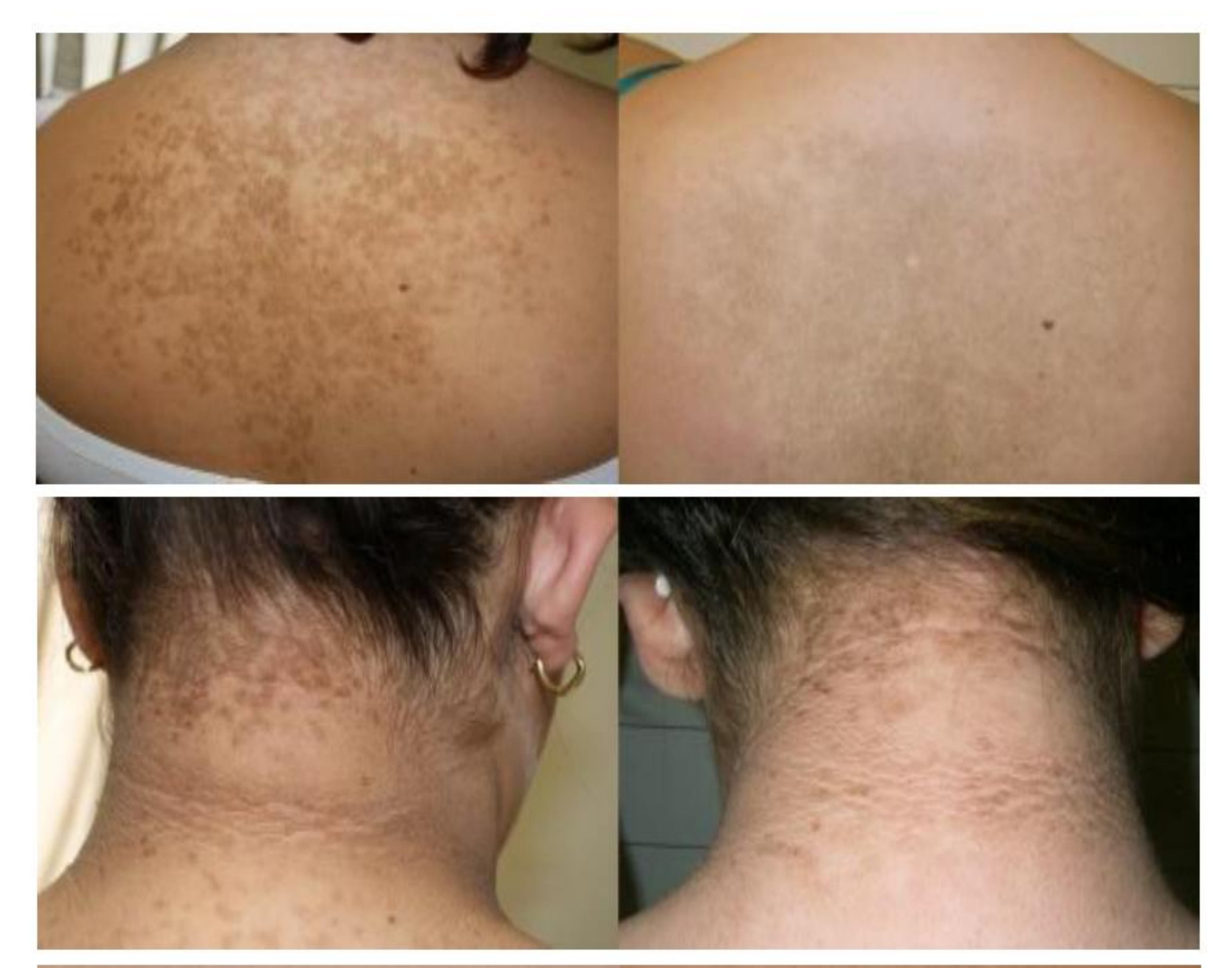

Table 2. Oral glucose tolerance tests (OGTT)

\section{References}

-Marcovecchio ML, Chiarelli F, (2014), An update on the pharmacotherapy options for pediatric diabetes, Expert Opin Biol Ther, Mar;14(3):355-64

-Brufani C et al, (2011), Use of metformin in pediatric age, Pediatr Diabetes, 2011 Sep;12(6):580-8

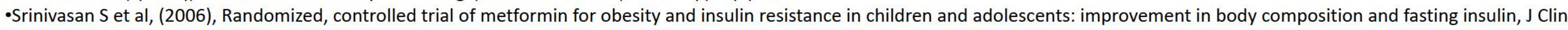
Endocrinol Metab, Jun;91(6):2074-80 